\title{
Kinematics of Polymer Chains in Dense Media. 3. Influence of Intramolecular Conformational Potentials
}

\author{
I. Bahar, ${ }^{*}+$ N. Baysal, ${ }^{\dagger}$ and B. Erman \\ Polymer Research Center and School of Engineering, Bogazici University, and TUBITAK \\ Advanced Polymeric Materials Research Center, Bebek 80815, Istanbul, Turkey
}

\section{Monnerie}

Ecole Supérieure de Physique et de Chimie Industrielles de Paris, PCSM, 10 rue Vauquelin, Cedex 05, Paris 75231, France

Received May 25, 1994; Revised Manuscript Received September 27, 1994

\begin{abstract}
The previously developed theory for treating the kinematics of polymers in dense media (Bahar, I.; Erman, B.; Monnerie, L. Macromolecules 1992, 25, 6309, 6315) is extended to include the influence of internal conformational energy barriers on the mechanism of motion. The method is based on the solution of a constrained equation of motion in the presence of dissipative forces due to friction. Successive solution of the equation for incremental changes in bond torsional angles up to completing one isomeric jump yields the optimal configurational rearrangements of chains of known original structure in response to bond isomerization. By repeating the method for an ensemble of Monte Carlo chains with different original conformations, the type and extent of coupling between dihedral angles, the correlation length involved in local conformational transitions, and the effective activation energies operating on a wide spectrum of viscous environments are determined as a function of the relative strength of intraand intermolecular effects. Comparison of results with those of Brownian dynamics simulations supports the adoption of the present model as a computationally efficient approach for investigating the kinematics of local motions in polymers.
\end{abstract}

\section{Introduction}

The mechanism and rate of conformational changes in polymer chains are mainly controlled by three factors: (i) chain connectivity, (ii) intramolecular conformational potential, and (iii) interactions with the environment.

The constraints imposed by chain connectivity operate on a wide spectrum of length scales, ranging from a few backbone bonds to Rouse type chain segments. The effect of connectivity is manifested either by favoring coupled bond rotations that highly localize the chain movement or by slowing down the isolated motions which are not accompanied by short-range compensating motions and thus would give rise to large-amplitude displacements of chain segments. ${ }^{1}$

The internal conformational potential of the chain may arise from three sources: bond stretching, distortion of bond angles, and torsional rotations of bonds. Among these, the latter constitutes the softest degree of freedom and controls to a large extent the relaxation mechanism. In fact, models with holonomic constraints, in which bond lengths and bond angles are constrained to assume fixed values, have found widespread use in treating polymer dynamics. Thus, the short-range internal resistance to motion refers in a broad sense to conformational energy barriers opposing the rotational isomeric transitions or torsional fluctuations of backbone bonds.

The interactions with the environment are of either intermolecular or long-range intramolecular origin. The frictional resistance to motion belongs to this category and will be taken up in the present study.

\footnotetext{
${ }^{+}$Permanent address: Department of Chemical Engineering, Bogazici University, Bebek 80815, Istanbul, Turkey.

${ }^{8}$ Abstract published in Advance ACS Abstracts, November 15 , 1994.
}

In a recently developed formalism treating the kinematics of conformational transitions in polymers, ${ }^{2-4}$ the minimization of the cumulative square displacements of chain units has been adopted as a fundamental criterion for controlling the mechanism of local motions, irrespective of intramolecular conformational energetics. The methodology inherently incorporated the effects of chain connectivity, whereas the internal resistance to rotameric transitions arising from the specific chemical and structural characteristics of the chain was not regarded. In this respect, the model chain was the freely rotating chain of polymer statistics, ${ }^{5}$ in which bond angles and bond lengths are fixed and bond torsions are not hindered by any potential. The optimal response of the chain to perturbations in dihedral angles has been obtained in these model chains, demonstrating the tendency of backbone bonds to undergo coupled counterrotations and/or small-amplitude fluctuations localizing the motion. Interestingly, several characteristics of local motions, which are generally extracted from the statistical analysis of long trajectories generated by Brownian dynamics (BD) simulations, ${ }^{6-9}$ were readily obtainable by the theory. Mainly, the types of the most frequent coupled rotations of backbone bonds and the distribution of the angular and translational displacements of chain units in response to a local perturbation conform closely with results from simulations. Yet, a more refined treatment should consider the specific chemical nature and conformational characteristics of the polymer. The aim of the present work is to extend the previous formalism so as to include the effect of torsional barriers on the kinematics of the motion.

The model and the basic formalism are described in the next section. The mathematical formulation of the theory is presented in the third section, which is followed by a section on calculations and discussion. Concluding remarks are presented in the last section. 

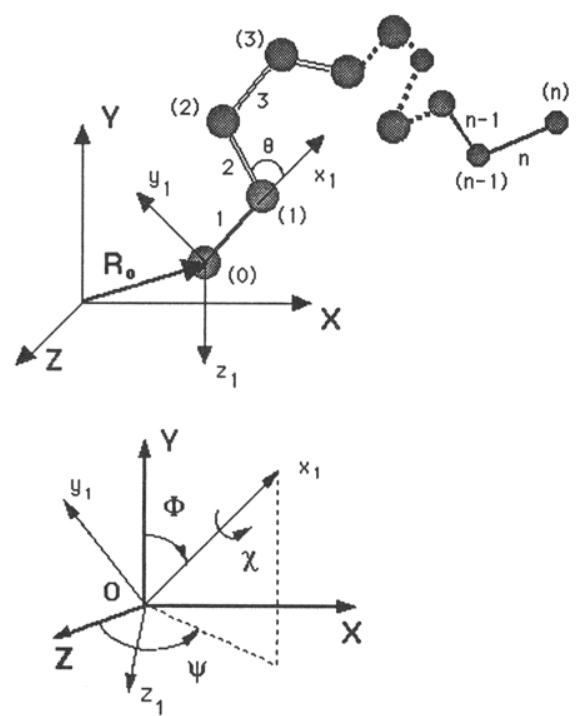

Figure 1. (a) A random configuration of a chain. The atoms are numbered from 0 to $n$ in the laboratory-fixed frame, $O X Y Z$. The first bond based coordinate system $x_{1} y_{1} z_{1}$ is shown. In general, the $x_{i}$ axis of the $i$ th bond-based frame is in the direction of the $i$ th bond. The $y_{i}$ axis is in the plane of the $i$ th and $(i-1)$ st bonds and makes an acute angle with the extension of bond $i-1$. The $z_{i}$ axis completes the right-handed frame. $\theta$ is the supplemental bond angle. In general, $\mathbf{R}_{i}$ and $\mathbf{r}_{i}$ denote the position vectors of atom $i$ in frames $O X Y Z$ and $x_{1} y_{1} z_{1}$, respectively. Here only $\mathbf{R}_{0}$ is shown. (b) Absolute orientation of the chain in space, identified by that of the first bond. $\Phi$ and $\Psi$ are the polar and azimuthal angles for the orientation with respect to the coordinate system OXYZ. The third Euler angle $\chi$ is identified with the torsional angle $\varphi_{1}$ for the first bond.

\section{Model and Basic Formalism}

Model. A polymer chain of $n$ backbone bonds with fixed bond lengths, $l$, and fixed bond angles, $\theta$, is considered. Atoms are indexed from 0 to $n$. The coordinates of the atoms are expressed in the laboratoryfixed frame, $O X Y Z$, shown in Figure 1a. The bond dihedral angles $\varphi_{m}, 2 \leq m \leq n-1$, are assumed to be subject to independent rotational potentials. The total number of degrees of freedom of the chain amounts to $n+4, n-2$ of them being associated with internal bond rotations and the remaining six with the absolute position and orientation of the chain. The absolute position of atom $i$ in frame $O X Y Z$ is denoted as $\mathbf{R}_{i}$. Accordingly, $\mathbf{R}_{0} \equiv \operatorname{col}\left[X_{0} Y_{0} Z_{0}\right]$ is the position vector of the zeroth atom. The absolute orientation of the chain in space is defined by the Euler angles $\Phi, \Psi$, and $\chi$ shown in Figure 1b. For convenience, the Euler angle $\chi$ will be identified with the torsional angle $\varphi_{1}$ of the first bond, and consequently the index of bond dihedral angles will be varied in the range $1 \leq m \leq n-1$. Thus, the set of generalized coordinates $\mathbf{q}=\left\{\varphi_{1}, \varphi_{2}, \ldots, \varphi_{n-1}\right.$, $\left.\Phi, \Psi, X_{0}, Y_{0}, Z_{0}\right\} \equiv\left\{q_{1}, q_{2}, \ldots, q_{n+4}\right\}$ will be adopted for describing the instantaneous configuration of the chain. The time derivative of the same set defines the ensemble of generalized velocities $\dot{q}$, whereas the Cartesian components of the velocity of particle $i$ is denoted as $\mathbf{v}_{i}$.

Basic Approach. In a medium with effective friction coefficient $\xi$, the motion of the $i$ th atom will be opposed by a frictional force $\mathbf{F}_{\xi_{i}}$ of the form

$$
\mathbf{F}_{\xi_{i}}=-\xi \mathbf{v}_{i} \equiv-\nabla_{\mathbf{v}_{i}} \mathscr{F}
$$

Here $\nabla_{\mathbf{v}_{i}}$ denotes the gradient with respect to $\mathbf{v}_{i}$ and $\mathscr{F}$ is Rayleigh's dissipation function defined as ${ }^{10}$

$$
\mathscr{F}=(1 / 2) \xi \sum_{i} \mathbf{v}_{i} \cdot \mathbf{v}_{i}=(1 / 2) \xi \sum_{i} \sum_{j} A_{i j} \dot{\mathbf{q}}_{i} \cdot \dot{\mathbf{q}}_{j}
$$

The first summation in eq 2 is performed over all atoms of the chain. $A_{i j}$ is the $i j$ th element of the operator transforming from the generalized coordinate system to the Cartesian system as will be discussed below.

The Lagrange equations of motion in the presence of frictional forces is expressed in terms of the dissipation function and the Lagrangian $L$ as $^{10}$

$$
\frac{\mathrm{d}}{\mathrm{d} t}\left[\frac{\partial L}{\partial \dot{q}_{j}}\right]-\frac{\partial L}{\partial q_{j}}+\frac{\partial \mathscr{F}}{\partial \dot{q}_{j}}=0
$$

The Lagrangian is given as $L=T-V$, where $T$ and $V$ are the kinetic and potential energies, respectively. $V$ being a state function, i.e., independent of velocities, the leading term of eq 3 reduces to the acceleration contribution. In a highly viscous medium, the contribution of this term to the equation of motion, as well as that of the gradient of $T$ with respect to the generalized coordinates, which are both proportional to the mass $m$ of the particles, may be neglected as a first approximation. This simplification is justifiable by the fact that the ratio $\mathrm{m} / \xi$ is commonly of the order of $10^{-14}-10^{-13}$ $\mathrm{s}$, and consequently these contributions are relatively small unless motions occurring in this high-frequency regime are of direct interest. ${ }^{11}$ Within the limits of this approximation, the Lagrange equation reduces to

$$
\frac{\partial V}{\partial q_{j}}+\frac{\partial \mathscr{F}}{\partial \dot{q}_{j}}=0
$$

Thus, the equation of motion is mainly governed by two contributions: the change in potential energy with respect to position in space, and the forces due to the frictional resistance of the surroundings.

The differential work $d W_{\xi_{i}}$ done by the $i$ th atom against friction reads

$$
\mathrm{d} W_{\xi_{i}}=-\mathbf{F}_{\xi_{i}} \cdot \mathrm{d} \mathbf{R}_{i}=\nabla_{\mathbf{v}_{i}} \mathscr{F} \cdot \mathbf{v}_{i} \mathrm{~d} t
$$

which leads to the expression

$$
\mathrm{d} W_{\xi} \equiv \sum_{i} \mathrm{~d} W_{\xi_{i}}=2 \mathscr{F} \mathrm{d} t=\xi \sum_{i} \mathbf{v}_{i} \cdot \mathbf{v}_{i} \mathrm{~d} t
$$

for the total differential work done by the chain against friction. For small incremental displacements $\delta \mathbf{R}_{i}=$ $\mathbf{v}_{i} \delta t$ of backbone atoms occurring within short time intervals $\delta t$, eq 6 may be approximated by

$$
\delta W_{\xi}=2 \mathscr{F} \delta t=(\xi / \delta t) \sum_{i} \delta \mathbf{R}_{i} \cdot \delta \mathbf{R}_{i}
$$

This term represents the energy dissipation due to friction during a differential change in configuration, taking place during the time increment $\delta t$. An explicit expression for the last summation in eq 7 in terms of the differential changes in generalized coordinates is readily obtained by combining eqs 6 and 18 of ref 2 . Substitution of this term in eq 4 yields

$$
\frac{1}{2} \frac{\partial \delta W_{\xi}}{\partial \delta q_{j}}+\frac{\partial V}{\partial q_{j}}=0
$$

Equation 8 directly follows from the identities $\mathscr{F}=(1 /$ 2) $\delta W_{\xi} / \delta t$ and $\dot{\mathbf{q}}=\delta \mathbf{q} / \delta t$. In the absence of a potential $V$, eq 8 becomes equivalent to the minimization of $\delta W_{\xi}$ with respect to the incremental variations in the 
generalized coordinates. This reduced form is in fact identical to the basic equation adopted in the previous treatment ${ }^{2}$ of the kinematics of a freely rotating chain model, following an externally imposed perturbation of an original configuration. The compromise between the cumulative square displacements of chain units and the rotational barriers to motion during the steepest descent path of reaction coordinate was also invoked by Skolnick and Helfand in their multidimensional extension of Kramers rate theory to conformational kinetics. ${ }^{12}$

Following the above description, the energy change associated with conformational transitions in a model chain with holonomic constraints would result from two contributions: (i) the energy spent to overcome the frictional resistance to motion and (ii) the change in conformational potential of the chain. The former is the work done by the chain against friction during the displacement of the atoms, and thus is path-dependent. The second is a state function and therefore depends on the initial and final configurations. In the most general case it includes both intra- and intermolecular effects. Here, the intramolecular effects are manifested by holonomic constraints and rotational potentials of the bonds. Intermolecular effects which are generally accounted for by explicit Lennard-Jones potentials or indirectly through a white noise will not be explicitly included in $V$. Instead, external constraints in the form of incremental perturbations of generalized coordinates will be adopted. Thus, the conformational potential $V$ of the chain will be given by

$$
V=\sum_{i=2}^{n-1} V_{\varphi}\left(\varphi_{i}\right)
$$

where $V_{\varphi}\left(\varphi_{i}\right)$ refers to the torsional energy of the $i$ th bond along the chain. Independence of bond torsional states is implicit in eq 9 . Following the expression proposed by Ryckaert and Bellemans for alkanes, $V_{\varphi}\left(\varphi_{i}\right)$ is taken as a fifth-order polynomial of the form ${ }^{13}$

$$
V_{\varphi}\left(\varphi_{i}\right)=k_{\varphi} \sum_{m=0}^{5} a_{m} \cos ^{m} \varphi_{i}
$$

where $k_{\varphi}$ is a proportionality constant and the coefficients $a_{0}, \ldots, a_{5}$ are $1.0,1.3100,-1.4140,-0.3297$, 2.8280 , and -3.3943 , respectively.

Using the above expressions for $V$ and $\delta W_{\xi}$, eq 8 reads in explicit notation in terms of the generalized coordinates

$$
\begin{gathered}
\frac{1}{2} \frac{\partial \delta W_{\xi}}{\partial \delta \varphi_{i}}+\frac{\partial V}{\partial \varphi_{i}}=0 \\
\frac{\partial \delta W_{\xi}}{\partial \delta \Phi}=\frac{\partial \delta W_{\xi}}{\partial \delta \Psi}=\frac{\partial \delta W_{\xi}}{\partial \delta \mathbf{R}_{0}}=0
\end{gathered}
$$

The subscript $i$ in eq 11 varies in the range $1 \leq i \leq n-$ 1. The potential $V$, being a function of internal coordinates only, does not appear in the second line of eq 11 . The last equality refers to the three derivatives with respect to the components of $\delta \mathbf{R}_{0}$. Explicit expressions for the derivatives of $\delta W_{\xi}$ with respect to the generalized coordinates are presented in the Appendix.

\section{Mathematical Formulation}

Let us consider the rotational potential $V$ of the chain at a given instantaneous configuration $\mathbf{q}$. This potential may be expressed as a Taylor series expansion in the close neighborhood of $\mathbf{q}^{\circ}=\left\{\varphi_{1}{ }^{\circ}, \varphi_{2}{ }^{\circ}, \ldots, \varphi_{n-1}{ }^{\circ}, \Phi^{\circ}, \Psi^{\circ}\right.$, $\left.X_{0}^{\circ}, Y_{0}^{\circ}, Z_{0}^{\circ}\right\}$ as

$$
V=V_{0}+\sum_{i}\left[\frac{\partial V}{\partial \varphi_{i}}\right]_{\mathbf{q}^{\circ}} \Delta \varphi_{i}+\frac{1}{2} \sum_{i} \sum_{j}\left[\frac{\partial^{2} V}{\partial \varphi_{i} \partial \varphi_{j}}\right]_{\mathbf{q}^{\circ}} \Delta \varphi_{i} \Delta \varphi_{j}+\ldots
$$

For the particular form of $V$ given by eq 10 , the second derivative vanishes for $i \neq j$. Accordingly, the derivative of $V$ with respect to the dihedral angle $\varphi_{m}$ at configuration $\mathbf{q}$ reads

$$
\partial V / \partial \varphi_{m}=\left(\partial V / \partial \varphi_{m}\right)_{\mathbf{q}^{\circ}}+\left(\partial^{2} V / \partial \varphi_{m}{ }^{2}\right)_{\mathbf{q}^{\circ}} \Delta \varphi_{m}
$$

for small $\Delta \varphi_{m}$.

In the following the notation $\Delta q_{m}$ and $\Delta t$, instead of $\delta q_{m}$ and $\delta t$, will be adopted for small incremental changes in generalized coordinates and for the corresponding time intervals, respectively. Also, the work performed at each step will be denoted as $W_{\xi}$, and the accompanying change in rotational potential will be indicated as $\Delta V=V-V_{0}$. This notation conforms with previous treatment as well as with the usual numerical method of solution of the equations of motion. It would be implicit in the notation below that all incremental changes refer to differential variations consistent with the basic approach.

Inasmuch as $\partial V / \partial \varphi_{m}=\partial \Delta V / \partial \Delta \varphi_{m}$, the first line of eq 11 may be rewritten in the form

$$
\frac{\partial\left[W_{\xi} / 2+\Delta V\right]}{\partial \Delta \varphi_{i}} \equiv \frac{\partial \Delta E}{\partial \Delta \varphi_{i}}=0
$$

in which the definition $\Delta E=W_{\xi} / 2+\Delta V$ has been introduced. Equations 13 and A-3 are inserted into eq 14 to obtain the equality

$$
\begin{aligned}
& \sum_{j=1}^{n-1}\left(u_{m j}+V_{m j}^{\prime \prime}\right) \Delta \varphi_{j}+p_{m} \Delta \psi+ w_{m} \Delta \Phi+ \\
& \mathbf{v}_{m} \cdot \Delta \mathbf{R}_{0}+V_{m}^{\prime}=0
\end{aligned}
$$

where $u_{m j}, p_{m}, w_{m}$, and $\mathbf{v}_{m}$ are defined in the Appendix. $V_{m}^{\prime}$ and $V_{m j}^{\prime \prime}$ are defined as

$$
\begin{gathered}
V_{m}^{\prime} \equiv(\delta t / \xi)\left(\partial V / \partial \varphi_{m}\right)_{\mathbf{q}^{\circ}} \\
V_{m j}^{\prime \prime} \equiv(\delta t / \xi)\left(\partial^{2} V / \partial \varphi_{m}{ }^{2}\right)_{\mathbf{q}^{\circ}} \delta_{m j}
\end{gathered}
$$

where $\delta_{m j}$ is the Kronecker delta equal to unity for $m=$ $j$ and zero otherwise.

If bond $s$ externally constrained to undergo a given rotational perturbation $\Delta \varphi_{s}$, eq 15 becomes

$$
\begin{array}{r}
\sum_{\substack{j=1 \\
j \neq s}}^{n-1}\left[u_{m j}+V_{m j}^{\prime \prime}\right] \Delta \varphi_{j}+p_{m} \Delta \Psi+w_{m} \Delta \Phi+\mathbf{v}_{m} \cdot \Delta \mathbf{R}_{0}= \\
-V_{m}^{\prime}-u_{m s} \Delta \varphi_{s}
\end{array}
$$

The subscript $\mathbf{q}^{\circ}$ in the gradient of potential on the right-hand side of the equality has been omitted for brevity. Likewise, the counterpart of the three equalities in the second line of eq 11 in the presence of the constraint $\Delta \varphi_{s}$ are 


$$
\begin{aligned}
\sum_{\substack{m=1 \\
m \neq s}}^{n-1} p_{m} \Delta \varphi_{m}+ & \sum_{i=0}^{n}\left(\mathbf{D} \mathbf{r}_{i} \cdot \mathbf{D} \mathbf{r}_{i}\right) \Delta \Psi+\sum_{i=0}^{n}\left(\mathbf{D} \mathbf{r}_{i} \cdot \mathbf{B} \mathbf{r}_{i}\right) \Delta \Phi+ \\
& {\left[\mathbf{T}(\Psi) \mathbf{T}(\Phi) \sum_{i=0}^{n} \mathbf{D} \mathbf{r}_{i}\right] \cdot \Delta \mathbf{R}_{0}=-p_{s} \Delta \varphi_{s} } \\
\sum_{\substack{m=1 \\
m \neq s}}^{n-1} w_{m} \Delta \varphi_{m}+ & \sum_{i=0}^{n}\left(\mathbf{B r}_{i} \cdot \mathbf{B} \mathbf{r}_{i}\right) \Delta \boldsymbol{\Phi}+\sum_{i=0}^{n}\left(\mathbf{D} \mathbf{r}_{i} \cdot \mathbf{B} \mathbf{r}_{i}\right) \Delta \Psi+ \\
& {\left[\mathbf{T}(\Psi) \mathbf{T}(\boldsymbol{\Phi}) \sum_{i=0}^{n} \mathbf{B r}_{i}\right] \cdot \Delta \mathbf{R}_{0}=-w_{s} \Delta \varphi_{s} }
\end{aligned}
$$

and

$$
\begin{aligned}
& \sum_{\substack{m=1 \\
m \neq s}}^{n-1} \mathbf{v}_{m} \Delta \varphi_{m}+\left[\mathbf{T}(\Psi) \mathbf{T}(\Phi) \sum_{i=0}^{n} \mathbf{D} \mathbf{r}_{i}\right] \Delta \Psi+ \\
& \quad\left[\mathbf{T}(\Psi) \mathbf{T}(\Phi) \sum_{i=0}^{n} \mathbf{B r}_{i}\right] \Delta \Phi+(n+1) \Delta \mathbf{R}_{0}+-\mathbf{v}_{s} \Delta \varphi_{s}
\end{aligned}
$$

$\mathbf{B}$ and $\mathbf{D}$ in eqs 18 and 19 are defined in the Appendix. Equations 17-20 are the basic equations of motion to be solved simultaneously to determine the new set of generalized coordinates following the perturbation of the original configuration $\mathbf{q}^{\circ}$. This set of equations may be conveniently represented in matrix notation as

$$
\left[\begin{array}{ll}
\mathbf{Q}_{1} & \mathbf{Q}_{2} \\
\mathbf{Q}_{2}{ }^{\mathrm{T}} & \mathbf{Q}_{\mathbf{4}}
\end{array}\right]\left[\begin{array}{l}
\Delta \varphi \\
\Delta \mathbf{X}
\end{array}\right]=\left[\begin{array}{l}
\Delta \varphi^{\circ} \\
\Delta \mathbf{X}^{\circ}
\end{array}\right]
$$

in which the solution is expressed in the form of an array of incremental changes in generalized coordinates, using the notation

$$
\Delta \varphi=\left[\begin{array}{lllllll}
\Delta \varphi_{1} & \Delta \varphi_{2} & \ldots & \Delta \varphi_{s-1} & \Delta \varphi_{s+1} & \ldots & \Delta \varphi_{n-1}
\end{array}\right]^{\mathrm{T}}
$$

and

$$
\Delta \mathrm{X}=\left[\begin{array}{lllll}
\Delta \Psi & \Delta \Phi & \Delta X_{0} & \Delta Y_{0} & \Delta Z_{0}
\end{array}\right]^{\mathrm{T}}
$$

$\Delta \varphi^{\circ}$ and $\Delta \mathrm{X}^{\circ}$ depend on the rotational perturbation of the bond $s$ and on the rotational potentials and are given by the expressions

$$
\begin{aligned}
& \Delta \boldsymbol{\varphi}^{\circ}= \\
& -\left[\begin{array}{lllllll}
u_{1 s} & u_{2 s} & \ldots & u_{s-1, s} & u_{s+1, s} & \ldots & u_{n-1, s}
\end{array}\right]^{\mathrm{T}} \Delta \varphi_{s}- \\
& \\
& {\left[\begin{array}{lllllll}
V_{1}^{\prime} & V_{2}^{\prime} & \ldots & V_{s-1}^{\prime} & V_{s+1}^{\prime} & \ldots & V_{n-1}^{\prime}
\end{array}\right]^{\mathrm{T}}} \\
& \Delta \mathbf{X}^{\circ}=-\left[\begin{array}{lllll}
p_{s} & w_{s} & \mathbf{v}_{s}^{\mathrm{T}}
\end{array}\right]^{\mathrm{T}} \Delta \varphi_{s}
\end{aligned}
$$

The matrix $\mathbf{Q}_{1}$ in eq 21 is defined by

$$
\mathbf{Q}_{1}=\mathbf{U}+\mathbf{V}^{\prime \prime}
$$

where $\mathbf{U}$ and $\mathbf{V}^{\prime \prime}$ are the matrices whose elements are given by equations A-7 and 16, respectively. The order of each of these matrices is equal to $n-2$, corresponding to the dihedral angle $\Delta \varphi_{m}$, with $1 \leq m \leq n-1$, the $s$ th row and column being absent. Likewise the matrices $\mathbf{Q}_{2}$ and $\mathbf{Q}_{4}$ take the form

$$
\mathbf{Q}_{2} \equiv\left|\begin{array}{lll}
p_{1} & w_{1} & \mathbf{v}_{1}^{\mathrm{T}} \\
p_{2} & w_{2} & \mathbf{v}_{2}^{\mathrm{T}} \\
\cdots & \cdots & \\
p_{s-1} & w_{s-1} & \mathbf{v}_{s-1}^{\mathrm{T}} \\
p_{s+1} & w_{s+1} & \mathbf{v}_{s+1}{ }^{\mathrm{T}} \\
\cdots & \cdots & \\
\cdots & \cdots & \\
\cdots & \cdots & \\
p_{n-1} & w_{n-1} & \mathbf{v}_{n-1} \mathrm{~T}
\end{array}\right|
$$

and

$\mathbf{Q}_{4} \equiv$

$\left[\begin{array}{lll}\sum_{i=0}^{n} \mathbf{D r}_{i} \cdot \mathbf{D} \mathbf{r}_{i} & \ldots & \ldots \\ \sum_{i=0}^{n} \mathbf{D} \mathbf{r}_{i} \cdot \mathbf{B} \mathbf{r}_{i} & \sum_{i=0}^{n} \mathbf{B r}_{i} \cdot \mathbf{B r} \mathbf{r}_{i} & \ldots \\ \mathbf{T}(\Psi) \mathbf{T}(\boldsymbol{\Phi}) \sum_{i=0}^{n} \mathbf{D} \mathbf{r}_{i} & \mathbf{T}(\Psi) \mathbf{T}(\boldsymbol{\Phi}) \sum_{i=0}^{n} \mathbf{B r}_{i} & (n+1) \mathbf{E}\end{array}\right]$

$\Delta \varphi$ and $\Delta \mathbf{X}$ are determined from

$$
\left[\begin{array}{c}
\Delta \varphi \\
\Delta \mathbf{X}
\end{array}\right]=\left[\begin{array}{ll}
\mathbf{Q}_{1} & \mathbf{Q}_{2} \\
\mathbf{Q}_{2}{ }^{\mathrm{T}} & \mathbf{Q}_{4}
\end{array}\right]^{-1}\left[\begin{array}{c}
\Delta \varphi^{\circ} \\
\Delta \mathbf{X}^{\circ}
\end{array}\right]
$$

which readily follows from eq 21 .

\section{Results of Calculations and Discussion}

General Calculation Procedure. Original configurations are generated for tetrahedrally bonded chains of $n$ bonds, each of length $1.53 \AA$, by assigning equilibrium rotational states trans $(t)$, gauche $e^{+}\left(g^{+}\right)$, or gauche$\left(\mathrm{g}^{-}\right)$to bond dihedral angles using the Monte Carlo (MC) method. The dihedral angle of internal bond $s$ will be assumed to undergo an incremental change $\Delta \varphi_{s}$. The accompanying set of incremental changes in generalized coordinates, mainly bond dihedral angles and absolute position and orientation of the chain, are determined from eq 29.

It should be recalled that the perturbation of the original configuration $\Delta \varphi_{s}$ should be small enough for representing the change in configuration in terms of discrete steps. In order to follow the configurational change in the chain accompanying a bond rotational jump from one isomeric state to another, for example, one should apply the above procedure repetitively by imposing a series of successive small incremental changes on the dihedral angle of the rotating bond. A choice of $\Delta \varphi_{s}=3^{\circ}$ was observed to be adequate in previous work $(V=0)$, inasmuch as adoption of smaller increments at each step was found to yield practically the same results. Thus, the rotameric jump of $\Delta \varphi_{s}=120^{\circ}$ would be performed in 40 successive steps, i.e., successive solutions of eq 29 , after updating at each step the new starting configuration. The time interval $\Delta t$ corresponding to each step would be approximately equal to $\lambda_{t g}{ }^{-1 /}$ 40 in this case, $\lambda_{t g}$ being the mean isomerization rate of an internal bond.

Upon introduction of the rotational potential $V$, the adoption of smaller $\Delta \varphi_{s}$ values, such as $\Delta \varphi_{s} \leq 1.5^{\circ}$, was required to ascertain the applicability of the equation of motion, eq 4 or 11, in difference form. The work done and the potential energy were computed at each step 

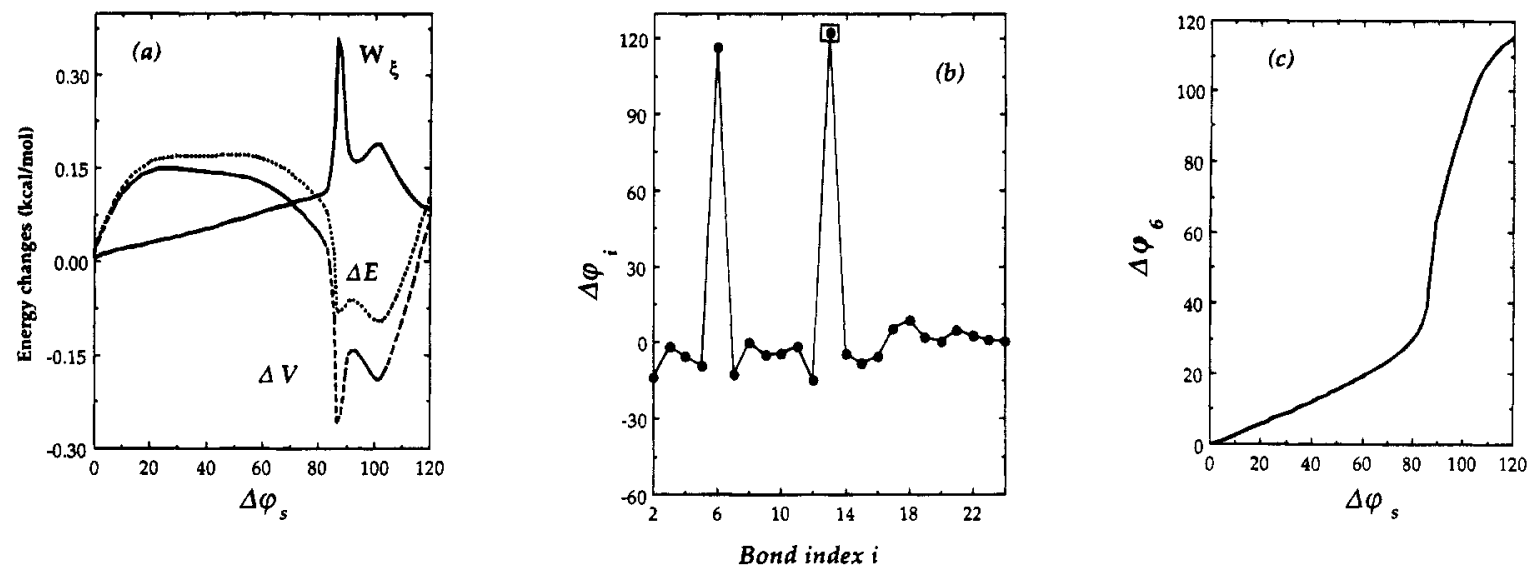

Figure 2. (a) Evolution of the work $W_{\xi}$ (solid curve) and change in rotational potential $\Delta V$ (dashed) of an example chain of 25 bonds during the rotameric transition of the middle bond between two isomeric states. The chain is originally in configuration $\left\{g^{+} g^{-} g^{-} t t t t g^{+} g^{-} g^{+} g^{-} g^{-} t g^{-} t g^{+} g^{+} t g^{-} t t g^{+} g^{-}\right\}$, and the central bond (boldface) undergoes the passage $g^{-} \rightarrow t$. W $W_{\xi}$ and $\Delta V$ represent the incremental work done and change in rotational energy, respectively, at successive dihedral angle increments of $\Delta \varphi_{s}=0.025$ $\mathrm{rad}$. $\xi / \Delta t$ is taken as $20 \mathrm{kcal} /\left(\mathrm{mol} \cdot \AA^{2}\right)$, and $k_{\varphi}=2 \mathrm{kcal} / \mathrm{mol}$. The dotted curve represents the change in the energy $\Delta E=W_{\xi} / 2+$ $\Delta V$ of the system during that particular rotameric transition. (b) Changes in the bond dihedral angles of the example chain of (a) following the rotational transition of the middle bond. The abscissa denotes the serial bond index, and the filled circles indicate the torsional rotations of the bonds relative to their original state. The rotation of the middle bond, which is externally imposed, is distinguished by a square enclosing the filled circle. (c) Evolution of the dihedral angle of the sixth bond of the example chain of (a) as a function of the rotation of the middle bond. The rotameric transition around $\Delta \varphi_{s}=90^{\circ}$ conforms with the energy changes observed in (a).

using eqs 7 and 9 , respectively, so as to verify whether the new state of the system conforms with the energy minimization principle implicit in eq 11. The size of $\Delta \varphi_{s}$ was rescaled by a factor of 2 , whenever necessary, on the basis of the incremental energy changes occurring in two successive steps. The prescription adopted for the readjustment of the step size at step $k$ on the basis of energy change occurring is as follows: If $\Delta E_{k+1}-\Delta E_{k}$ $>1.2\left(\Delta E_{k}-\Delta E_{k-1}\right)$, where the subscript $k$ refers to the calculation step, $\Delta \varphi_{\mathrm{s}}$ is divided by 2 . Alternatively, for the case $\Delta E_{k+1}-\Delta E_{k}<1.2^{-1}\left(\Delta E_{k}-\Delta E_{k-1}\right), \Delta \varphi_{s}$ is multiplied by 2 . The adoption of such variable step size increases the efficiency and the accuracy of the calculation.

The above procedure yields the optimal rearrangement of the chain for accommodating the rotational jump of a bond for a given initial configuration. For assessing the general response of internal and external coordinates to bond rotational transitions, a sufficiently large number of $\mathrm{MC}$ chains should be generated. This approach, which proved to be quite informative on the mechanism of the cooperative relaxation in the absence of internal conformational energies, ${ }^{3}$ will be undertaken below for chains subject to barriers hindering bond rotations.

An Ilustrative Example. It might be interesting to observe, as an example, the behavior of a randomly generated $\mathrm{MC}$ chain in response to a rotameric transition of an internal bond. We consider, for example, a chain of 25 bonds with the randomly chosen initial configuration $\left\{g^{+} g^{-} g^{-} t t t t g^{+} g^{-} g^{+} g^{-} g^{-} t g^{-} t g^{+} g^{+} t g^{-} t t g^{+} g^{-}\right\}$. The middle bond is constrained to rotate by a total amount of $\Delta \varphi_{s}=120^{\circ}$, i.e., from the $g^{-}$to $t$ state, through a succession of steps each smaller than $1.5^{\circ}$. The resulting changes in the generalized coordinates and consequently the conformational rearrangements of the chain accompanying the rotational transition of the middle bond $(s=13)$ are determined from successive solution of eq 29 at each incremental change $\Delta \varphi_{s}$.

The evolution of the rotational potential of the chain and that of the work done against friction throughout this particular rotational transition are displayed in
Figure 2a. The solid curve describes the work $W_{\xi}$ performed at fixed increments in the dihedral angle of the central bond; precisely, it is obtained by evaluating the differential work done by the chain for accommodating each successive torsional rotation of $\Delta \varphi_{s}=0.025 \mathrm{rad}$ (the latter being performed in more than one step whenever necessary, as delineated above), using eqs 7 in difference form with eqs $A-1$ and A-2, $\xi / \Delta t$ is taken as $20 \mathrm{kcal} /\left(\mathrm{mol} \cdot \AA^{2}\right)$. This value may be estimated as follows: for a dihedral angle increment of $0.025 \mathrm{rad}, \Delta t$ $\approx 0.025 \lambda_{t g}{ }^{-1} /(2 \pi / 3)=0.003 \mathrm{~ns}$, using an approximate $t$ $\rightarrow g$ isomerization rate $\lambda_{t g}$ of $4 / \mathrm{ns}^{6}{ }^{6}$ If the friction coefficient is taken as $2.5 \times 10^{4} \mathrm{~kg} /(\mathrm{mol} \cdot \mathrm{ns})$, which is 1 order of magnitude larger than that used for simulating dilute solutions of polyethylen $e^{6}$ and polyisoprene $e^{8}$ and thus corresponds to denser polymeric systems, we obtain $\xi / \Delta t=20 \mathrm{kcal} /\left(\mathrm{mol} \AA^{2}\right)$. The effect of various choices of friction coefficients will be further considered below. The dashed curve, labeled by the symbol $\Delta V$, describes the accompanying change in the rotational potential of the chain for $k_{\varphi}=2.0 \mathrm{kcal} / \mathrm{mol}$. A steady rise in $W_{\xi}$ is observed at the initial stages of the rotation of bond $s$, while at about $\Delta \varphi_{s}=90^{\circ}$ a sharp increase occurs, indicating at this point a relatively large displacement of atoms in space. At about the same point, the rotational potential of the chain exhibits a substantial drop, which would suggest the occurrence of an energetically favorable conformational change. The reduction in rotational energy more than counterbalances the work done against friction, as apparent from the resulting change in the total energy $\Delta E=W_{\xi} / 2+\Delta V$, which is displayed by the dotted curve.

Examination of the changes in bond dihedral angles, after the externally imposed $120^{\circ}$ rotation of the middle bond is completed, reveals in Figure $2 b$ that the response of the chain to this perturbation is mainly manifested by the rotational transition of a single bond, the sixth, while all other bonds closely preserve their original dihedral angles. In the figure the change in torsional angle of all bonds is indicated by filled circles except for that of the middle bond $s$, which is shown by a square in order to point out that this particular 
rotation is deliberately performed while others occur as a response. If one observes the evolution of the dihedral angle of the sixth bond, $\Delta \varphi_{6}$, in Figure $2 c$, an abrupt change or a rotameric jump is detected at about $\Delta \varphi_{s}=$ $90^{\circ}$, which conforms with the picture displayed in Figure $2 \mathrm{a}$.

This analysis provides a clear picture of the competition between intramolecular conformational energetics and intermolecular resistance to motion, which determines the precise mechanism of relaxation following an external perturbation. The exact response of a chain given to a perturbation of a generalized coordinate depends on its original configuration. A correlation between bonds 6 and 13 is demonstrated in this particular example. However, to infer some general information on the mechanism of local conformational motions or on the degree of correlations between bond rotational transitions, etc., a statistically large enough ensemble of MC chains with a variety of original configurations need to be analyzed, as will be undertaken below.

General Trends of MC Chains. For the analysis of the behavior of MC-generated chains, it will prove convenient to define a dimensionless ratio relating the strength of the two factors affecting the mechanism of motion, i.e., friction and rotational energy barriers. We may consider, for instance, the ratio

$$
k_{0}=\frac{2 k_{\varphi}}{l^{2} \xi / \Delta t}
$$

which, upon insertion into the first line of eq 11 together with eqs 7 and 9 leads to

$$
\frac{\partial}{\partial \Delta \varphi_{m}}\left[\sum_{i=0}^{n}\left(\Delta \mathbf{R}_{i}\right)^{2} / l^{2}+k_{0} \sum_{i=2}^{n-1} \sum_{m=0}^{5} a_{m} \cos ^{m} \varphi_{i}\right]=0
$$

From the examination of eq 31 , it is clear that the solution is uniquely obtained for fixed values of the ratio $k_{0}$ rather than the absolute values of the friction coefficient, the isomerization time, and the energy barrier for bond torsion. Thus, the results reported in this section are expressed as a function of the dimensionless ratio $k_{0}$. In principle, the passage to specific chains would be established by proper substitution of real parameters in eq 30 .

The following aspects of the kinematics of conformational transitions will be analyzed in the following: (i) the coupling between dihedral angles, (ii) the work and overall conformational energy changes accompanying bond torsional motions, and (iii) the distribution of angular and translational displacements of chain units in the neighborhood of a bond subject to a rotational isomerization. In general, sets of $2000 \mathrm{MC}$ chains with different original configurations have been considered for each choice of $k_{0}$ and $n$ in the following, and the constrained equation of motion (eq 29) has been successively solved for each incremental rotation of the middle bond until the completion of its isomeric transition in each MC chain. The theory has been applied to chains of length $n=25$ and 39 , subject to various strengths of frictional resistance and heights of rotational barriers.

(i) Coupling between Dihedral Angles. The changes in the dihedral angles in response to the rotational transition of a given bond, averaged over the ensemble of MC chains, are displayed in Figure 3. Figure $3 \mathrm{a}$ is obtained for $n=39$ and $k_{0} l^{2}=1 \mathrm{~A}^{2}$, with the rotating bond index $s=20$. The ensemble averages
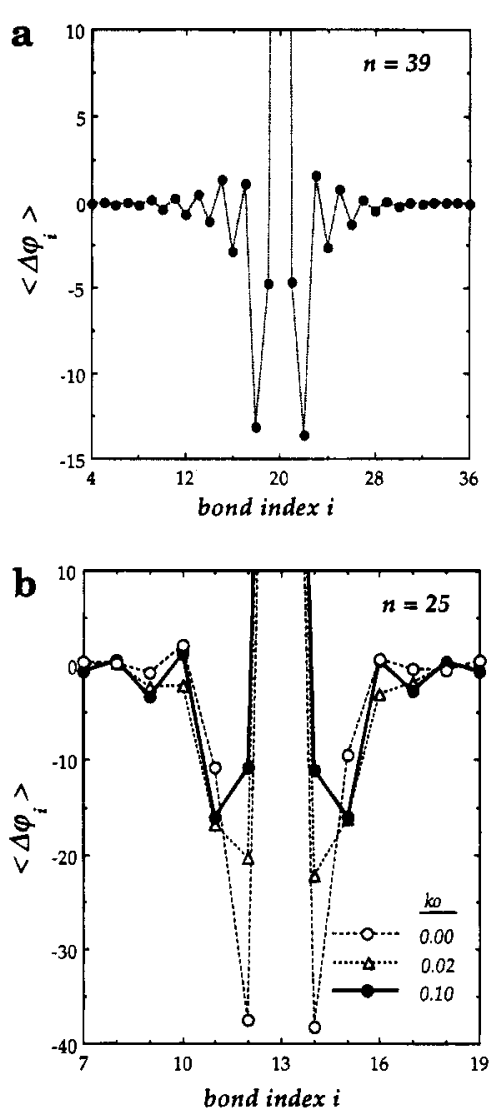

Figure 3. (a) Distribution of the average change, $\left\langle\Delta \varphi_{i}\right\rangle$, in bond dihedral angles in response to $120^{\circ}$ rotation of the middle bond evaluated from $2000 \mathrm{MC}$ chains of length $n=39$ presented as a function of bond index. Results are obtained from the solution of eq 31 (or eq 29) with $k_{0} l^{2}=1 \AA^{2}$. The rotation of the middle bond $s=20$ is performed in steps of $\Delta \varphi_{s} \leq 0.025 \mathrm{rad}$. (b) Average change, $\left\langle\Delta \varphi_{i}\right\rangle$, in bond dihedral angles induced by the $120^{\circ}$ rotation of the central bond of 2000 MC chains of length $n=25$ presented as a function of bond index. Results are obtained from the solution of eq 31 (or eq 29) with $k_{0} l^{2}=0,0.02$, and $0.10 \AA^{2}$, indicating that the response of the second neighbor is enhanced with increasing strength of internal barriers to rotation relative to frictional effects.

$\left\langle\Delta \varphi_{i}\right\rangle$ for $4 \leq i \leq 36$ are plotted against bond serial number $i$ and are connected by the dotted line to guide the eye. Two important observations are as follows: (i) Second neighbors along the chain exhibit the strongest response to bond rotation. A counterrotation of about $14^{\circ}$ is undergone by these bonds. First neighbors also respond in the same direction but their average change in torsional angle is smaller than that of second neighbors. (ii) The average changes in dihedral angles become vanishingly small beyond approximately sixth neighbors on both sides of the rotating bond. However, this does not necessarily imply that these dihedral angles remain unchanged but rather that they do not exhibit a tendency to rotate in a well-defined direction and hence cancel out in averaging over the whole set of MC chains. An assessment of the average amplitude of torsional motion of neighboring bonds, irrespective of the sense of rotation, will necessitate the examination of the average changes in the absolute values of dihedral angles $\left\langle\left|\Delta \varphi_{i}\right|\right\rangle$, as will be described below.

Before proceeding to the examination of $\left\langle\left|\Delta \varphi_{i}\right|\right\rangle$, we compare in Figure $3 \mathrm{~b}$ the $\left\langle\Delta \varphi_{i}\right\rangle$ results obtained for various $k_{0}$ values. It is clear from eq 30 that the dimensionless ratio $k_{0}$ increases with $k_{\varphi}$, i.e., with increasing strength of internal barriers to rotation relative to frictional effects, and becomes zero in the 


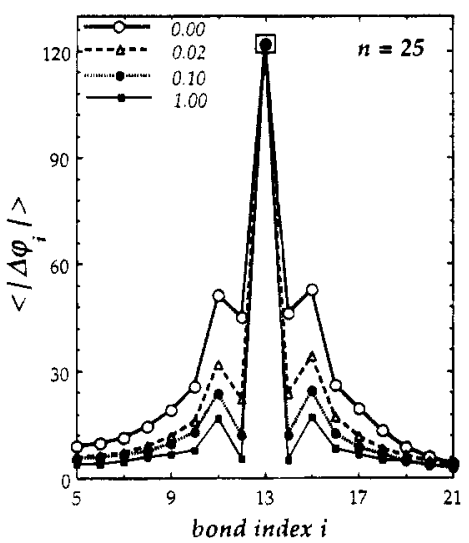

Figure 4. Average absolute change in bond dihedral angles following the rotation of the central bond as a function of bond index for $n=25$ and $k_{0} l^{2}=0,0.02,0.10$, and $1.0 \AA^{2}$.

absence of intramolecular rotational potentials. The results shown by the empty circles, empty triangles, and filled circles in Figure $3 \mathrm{~b}$ are obtained for $k_{0} l^{2}=0,0.02$, and $0.10 \AA^{2}$, respectively, and for $n=25$. When the motion is fully controlled by viscous effects $\left(k_{0}=0\right)$, we note that the first neighboring bond exhibits the strongest tendency to undergo a counterrotation, whereas upon introducing energy barriers opposing torsional rotations, the response of the second bond is gradually enhanced.

The average absolute changes $\left\langle\left|\Delta \varphi_{i}\right|\right\rangle$ in dihedral angles are presented as a function of bond index for $n$ $=25$ in Figure 4 . The results are displayed by the empty circles, empty triangles, filled circles, and filled squares for $k_{0} l^{2}=0,0.02,0.10$, and $1.0 \AA^{2}$, respectively. We note that the absolute variations in all dihedral angles diminish with increasing rotational barriers. The second neighbor exhibits the strongest change in dihedral angle in all cases, indicating that this is a general behavior for tetrahedrally bonded polyethylene-like chains, irrespective of the relative strength of intermolecular vs intramolecular effects. The only consequence of introducing intramolecular torsional energy barriers is the reduction of the amplitude of dihedral angle fluctuations in the close neighborhood of the rotating bond, without affecting the general shape of the distribution curves. In particular, the value $k_{0} l^{2}=1.0 \AA^{2}$ is representative of a system (see below) in which the internal barriers are significantly strong relative to friction effects. The dihedral angle fluctuations of the neighboring bonds in this case are strongly depressed. From the width of the distribution curves, we may infer that the torsional coupling between neighboring bonds extends up to approximately bonds $s \pm 4$, the remaining bonds being weakly affected by the transition of bond $s$. The agreement between shape of the present curves - and that resulting from BD simulations of polyethylene chains ${ }^{9}$ is noteworthy, particularly when considering the fact that the efficiency of the present method is significantly superior to that of simulations.

It should be emphasized that the curves displayed in Figure 4 are not restricted to $n=25$ only but are valid for chains of different lengths. In fact, calculations performed for $n=39$ demonstrated that indistinguishable curves are obtained with same data, provided that the abscissa is rescaled according to the rotating bond.

(ii) Energy Changes Accompanying Bond Torsions. The average change in the rotational potential of the overall chain and the average work done against friction during a bond rotameric transition have been evaluated. Parts a-c of Figure 5 display the evolution of the work done $\left(W_{\xi}\right)$ against friction, the overall rotational energy change $\left(\Delta V=V-V_{0}\right)$, and the total energy change $\left(\Delta E=W_{\xi} / 2+\Delta V\right)$, respectively, as a function of the change in the dihedral angle of the middle bond. The solid curves are obtained for $n=25$. For comparative purposes, results for $n=39$ are also shown by the dashed curves. Here, the curves represent the cumulative changes in the energy contributions; i.e., they are evaluated by summing up the changes in energy occurring at consecutive steps until the completion of the isomeric transition of $120^{\circ}$, in contrast to the curves of Figure $2 a$ in which the energy changes at each individual step of $\sim 1.5^{\circ}$ only were displayed. Results are shown for the four cases $k_{0} l^{2}=0,0.02,0.06$, and $0.10 \AA^{2}$, which are denoted by the respective labels a, $b, c$, and d on the figures. Curve $a$, which corresponds to the particular case $k_{0}=k_{\varphi}=0$ and consequently $\Delta V$ $=0$, is absent in Figure 5b. For providing an estimate of the magnitudes of energy changes accompanying bond rotations, the ordinate values corresponding to $\xi / \Delta t=$ $10 \mathrm{kcal} /\left(\mathrm{mol} \cdot \AA^{2}\right)$ are displayed in the figures. Thus, using eq 30 , curves $a-d$ in the figures correspond to rotational energy coefficients of $k_{\varphi}=0,0.1,0.3$, and 0.5 $\mathrm{kcal} / \mathrm{mol}$, respectively.

We observe in Figure 5a that the work done against environment increases with increasing $k_{0}$, i.e., increasing $k_{\varphi}$ or decreasing $\xi / \Delta t$, as follows from eq 30 . That the work done should increase with $k_{\varphi}$ is evident. As to the effect of $\xi / \Delta t$, one might also expect at first sight an increase in $W_{\xi}$ with $\xi / \Delta t$ upon considering eq 7 . However, it should be pointed out that with increasing environmental resistance, the displacements $\delta \mathbf{R}_{i}$ themselves are reduced, which more than counterbalances the effect of the front term $\xi / \Delta t$ in eq 7 .

The work done against environment, averaged over all $\mathrm{MC}$ chains, is found to increase smoothly during the rotameric transition of the central bond in Figure 5a, whereas the conformational energy of the overall chain exhibits in Figure $5 \mathrm{~b}$ a peak centered at about $\Delta \varphi_{s}=$ $60^{\circ}$. This peak exceeds the activation energy $\left(k_{\varphi}\right)$ for the rotational transition of a single bond in all cases, which indicates the simultaneous rotation and/or torsional fluctuations of one or more other bonds along the chain. Calculations repeated with $n=39$ for cases c and $\mathbf{d}$ are found to yield the changes in conformational energies shown by the dashed curves in Figure $5 \mathrm{~b}$. These curves are rather close to those (solid) obtained for $n=25$. Therefore, the observed change in conformational potential arises mainly from local rearrangements of the chain and is practically independent of molecular weight. Furthermore, the work contribution, displayed in Figure 5a by the dashed curves, also remains almost identical irrespective of chain length, confirming the local character of the motion.

The maxima in total energy change curves in Figure $5 \mathrm{c}$ may be identified as the effective activation energies for rotameric transition in various restrictive media. In all cases, this energy, $\Delta E_{\max }$, definitely exceeds that of a single-bond rotameric barrier. Even if the viscous contribution is subtracted from $\Delta E$, i.e., if attention is confined to the change in the overall conformational potential of the chain, shown in Figure 5b, the internal energy barrier $\left(V-V_{0}\right)_{\max }$ to be surmounted during the rotameric transition of bond $s$ is significantly larger than the activation energy for single-bond rotation. At first sight, this observation seems incompatible with the experimentally observed ${ }^{14}$ behavior of polymers in dilute 

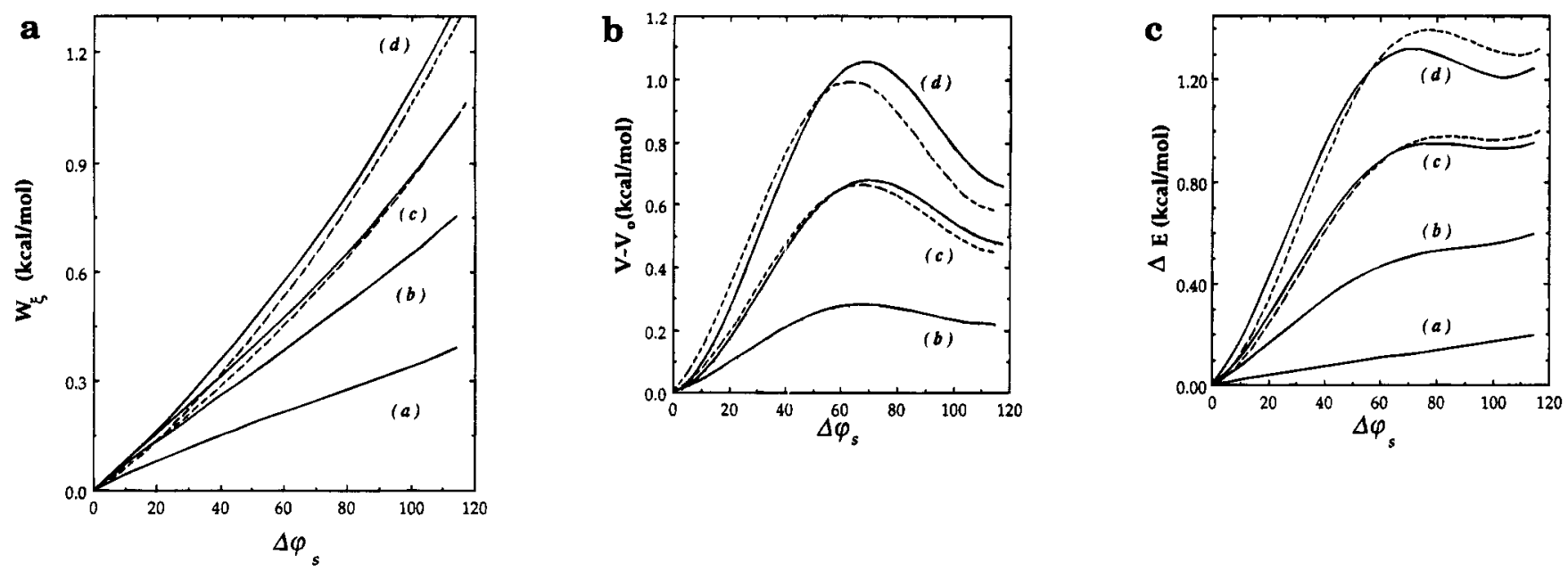

Figure 5. (a) Evolution of the work $W_{\xi}$ done by the chain against friction during the isomeric rotation of an internal bond averaged over the optimal path evaluated for $2000 \mathrm{MC}$ chains of different original configurations. The solid curves are obtained for $n=25$ and three dense media characterized by the parameters (a) $k_{0} l^{2}=0$, (b) 0.02 , (c) 0.06 , and (d) $0.10 \AA^{2}$ by summing up the work performed at successive steps. The dashed curves are obtained for the cases (c) and (d) with $n=39$. Their close similarity with the solid curves demonstrates that the observed motions are highly localized and independent of chain length. (b) Evolution of the average change in the conformational potential of the chain during the isomeric rotation of an internal bond. $V_{0}$ refers to the conformational potential of the chain prior to the rotameric transition of bond $s$. (c) Evolution of the total energy $\Delta E=W_{\xi} / 2$ $+\Delta V$ of the chain during the rotameric transition of an internal bond.

solution: the apparent activation energy for a given isomeric jump is of the order of a single-bond rotational energy barrier, which is also confirmed by theory. 1,15 However, the main object of the present study being to visualize the kinematics of macromolecules in dense media, the parameters in Figure 5a-c were intentionally selected so as to reproduce the effect of a rather restrictive environment, and the results hold for that particular situation.

If, on the other hand, the strength of the frictional effect is reduced relative to internal rotational barriers, by increasing the ratio $k_{0}$ for example, we may notice from Figure $5 \mathrm{~b}$ that the difference between $\left(V-V_{0}\right)_{\max }$ and the single-bond rotation barrier height $k_{\varphi}$ gradually diminishes. In fact, the ratio $\left(V-V_{0}\right)_{\max } / k_{\varphi}$ decreases approximately from 3.0 to 2.1 in Figure $5 \mathrm{~b}$ as $k_{0} l^{2}$ is varied from 0.02 to $0.1 \AA^{2}$. For an assessment of the behavior predicted by the theory as intermolecular effects are further weakened, calculations have been repeated for $k_{0} l^{2}=0.2,0.6$, and $1.0 \AA^{2}$. Curves $\mathrm{a}, \mathrm{b}$, and $\mathrm{c}$ in Figure $6 \mathrm{a}$ are obtained by taking $k_{\varphi}=1.0 \mathrm{kcal} /$ $\mathrm{mol}$ and $\xi / \Delta t=10.0,3.33$, and $2.0 \mathrm{kcal} /\left(\mathrm{mol} \cdot \AA^{2}\right)$, respectively. The energy barrier $\left(V-V_{0}\right)_{\max }$ for the overall conformational rearrangement of the chain gradually approaches the activation energy $\left(k_{\varphi}=1 \mathrm{kcal} / \mathrm{mol}\right)$ for single isolated rotation as $\xi / \Delta t$ decreases. In spite of the accompanying increase in displacements, the change in the overall energy $\Delta E$ exhibits the same tendency as $\Delta V$ as well, as shown in Figure $6 \mathrm{~b}$, which corroborates that an apparent activation energy of about a singlebond rotational barrier is involved during local conformational rearrangements of polymers in less restrictive environments such as that in dilute solution.

(iii) Angular and Translational Displacements of Chain Units. Close neighboring bonds along the chain undergo the spatial reorientations displayed in Figure 7 in response to the rotational transition of an internal bond. Curves $a, b$, and $c$ in the figure hold for $k_{0} l^{2}=0,0.02$, and $0.10 \AA^{2}$, respectively. The abscissa denotes the bond location relative to the rotating bond. Results for $n=25$ and 39 are found to be almost indistinguishable. The reorientation of the first neighboring bonds in space is the largest $\left(\sim 55^{\circ}\right)$, as would be
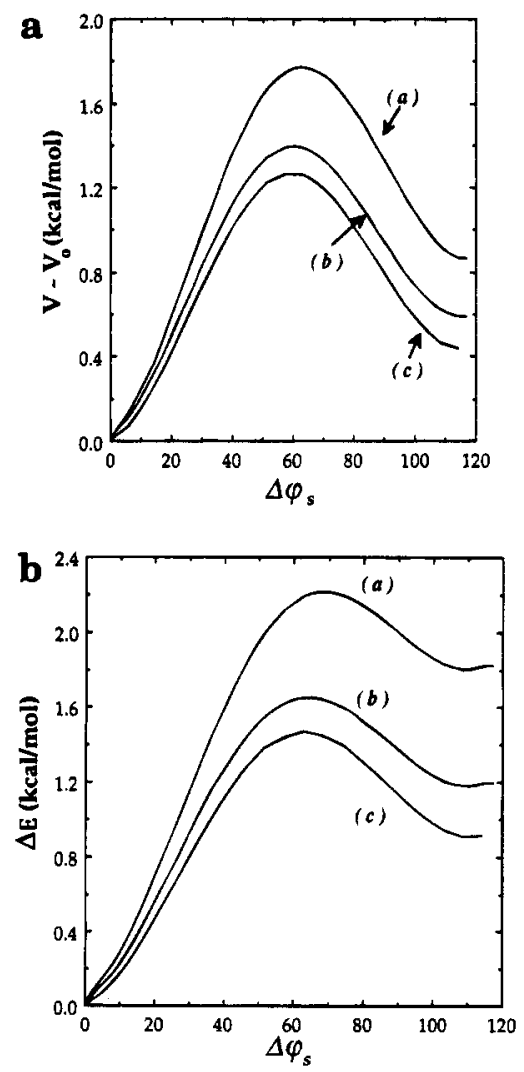

Figure 6. (a) Average change in the overall conformational potential of the chain, $V-V_{0}$, accompanying a bond rotational transition at three different environments given by (a) $\xi / \Delta t=$ 10.0 , (b) $\xi / \Delta t=3.33$, and (c) $\xi / \Delta t=2.0 \mathrm{kcal} /\left(\mathrm{mol} \cdot \AA^{2}\right)$. The curves are obtained for $n=25$ and $k_{\varphi}=1.0 \mathrm{kcal} / \mathrm{mol}$. Compared to Figure $5 \mathrm{a}-\mathrm{c}$, the present choice of parameters corresponds to relatively weaker frictional resistance and/or stronger internal barriers. (b) Average energy change $\Delta E=$ $W_{\xi} / 2+\Delta V$ accompanying bond rotational transition for the model chains $a, b$, and c displayed in (a). The activation energy for the overall conformational change, estimated from the maxima in the curves, approaches that of single-bond rotation $k_{\varphi}=1.0 \mathrm{kcal} / \mathrm{mol}$, with decreasing $\xi / \Delta t$.

expected from the symmetric distribution of the torsion of $120^{\circ}$ on both sides of the rotating bond. The angular 


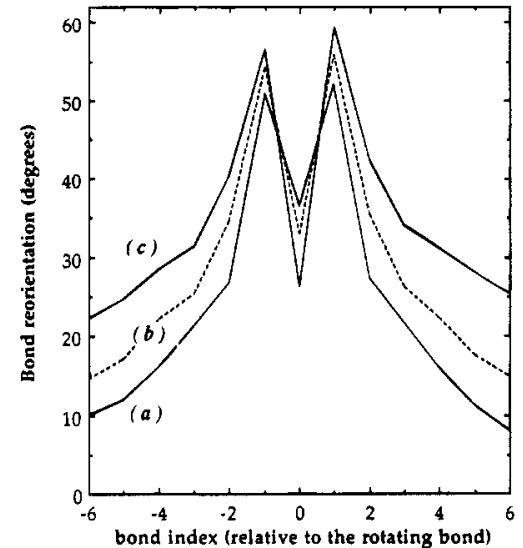

Figure 7. Spatial reorientation of bonds in the neighborhood of the bond undergoing the rotational transition. Bond indices on the abscissa are given relative to the rotating bond. Ordinate values represent the mean changes in the absolute orientation of bonds in space at the end of the isomeric transition of the middle bond. Results are obtained for (a) $k_{0} l^{2}$ $=0$, (b) $k_{0} l^{2}=0.02 \AA^{2}$, and (c) $k_{0} l^{2}=0.10 \AA^{2}$.

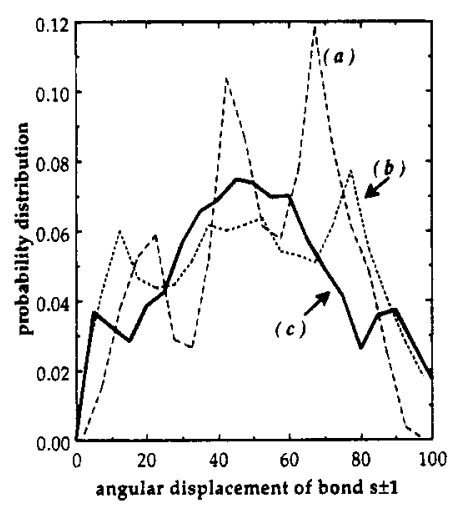

Figure 8. Probability distribution of angular displacement of bonds $s \neq 1$ in response to the rotameric transition of bond $s$ for (a) $k_{0} l^{2}=0$, (b) $k_{0} l^{2}=0.02 \AA^{2}$, and (c) $k_{0} l^{2}=0.10 \AA^{2}$. The distribution curves become smoother with increasing internal barriers and/or decreasing frictional resistance, although the broad character and the three peaks persist.

displacement of the near neighboring bonds is found to increase with $k_{0}$, i.e., in the presence of higher internal barriers to torsion and/or weaker frictional resistance.

Although the mean angular displacement of bonds $s$ \pm 1 is $\sim 55^{\circ}$, a more detailed examination of angular displacements of bonds $s \pm 1$ has revealed in previous work $^{3}$ the occurrence of a relatively broad distribution, with three peaks located for the particular case $k_{0}=0$. This result is reproduced in Figure 8, curve a, for comparative purposes. Calculations performed with $k_{0} l^{2}$ $=0.02$ and $0.10 \AA^{2}$ yield distribution curves $b$ and $c$, respectively, in Figure 8 . Thus, the broad character of the distribution is preserved and even enhanced in the presence of internal barriers opposing bond torsional motions. Three peaks are still observable, although the distribution is now rendered smoother with the introduction of internal barriers, competing with external resistance to motion. This wide variety of angular displacements induced by bond rotational transitions suggests that the small-amplitude angular distortions observed in 2-d NMR experiments ${ }^{16}$ should not be necessarily attributed to the absence of rotameric jumps but might be viewed as a manifestation of the cooperative response of several units along the chain to bond rotational transitions.

The mean displacement of atoms in response to a bond rotation is shown in Figure 9 as a function of

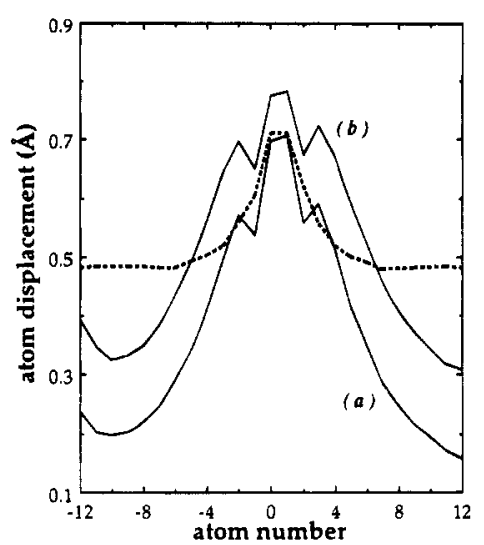

Figure 9. Average spatial displacements, $\left\langle\Delta \mathbf{R}_{i}\right\rangle$ of the chain atoms in response to the rotameric transition of the central bond given as a function of their atom index. The curves are obtained for (a) $k_{0} l^{2}=0.02$ and (b) $0.10 \AA^{2}$ with $n=25$. The dashed curve represents the results of Adolf and Ediger, ${ }^{17}$ obtained by BD simulations of polyethylene chains.

atomic position relative to the rotating bond. The latter is assumed to be composed of atoms 0 and 1 . The curves are obtained for (a) $k_{0} l^{2}=0.02$ and (b) $k_{0} l^{2}=0.10 \AA^{2}$ with $n=25$. For comparative purposes, the result of Adolf and Ediger ${ }^{17}$ obtained by $\mathrm{BD}$ simulations of polyethylene chains is displayed by the dashed curve. The displacement of atoms 0 and 1 is of comparable magnitude $(\sim 0.7 \AA)$ in the two approaches as well as that of the first three neighboring atoms on both sides. However, there is a clear deviation between our results and those from simulations as to the displacement of atoms located beyond fourth neighbors of the rotating bond. The theory predicts a gradual leveling off of atomic displacements as the atoms get farther apart from the rotating bond, whereas $\mathrm{BD}$ simulations yield a plateau value of about $0.48 \AA$. Calculations repeated with $n=39$ for various $k_{0} l^{2}$ also indicate a correlation length extending up to \pm 8 neighbors along the chain, whereas the remaining atoms exhibit mean displacements below $0.1 \AA$, except for a few terminal atoms which enjoy a higher degree of freedom. Thus, the conformational rearrangements induced by a bond rotameric jump is shown to be localized in the present work. The existence of a plateau value in BD simulations, on the other hand, is attributed to the presence of a continuous noise in the system. The observed displacement in simulations results from the superposition of a multitude of relaxation processes induced by rotameric jumps taking place at various loci, whereas in our present case the noise arising from the simultaneous rotation of other bonds driven by external effects is totally eliminated. We note, furthermore, that the second neighboring atom in model chains of $n=25$ have a tendency to undergo relatively large displacements, compared to first neighboring atoms. However, additional calculations with $n=39$ and $k_{0} l^{2}=0.02 \AA^{2}$ indicate that this feature does not persist in longer model chains subjected to stronger environmental effects, in agreement with the distribution obtained by BD simulations.

\section{Concluding Remarks}

In the present work, a new method has been developed for determining the unique response of a given chain of known configuration in a dense environment to a specific perturbation. The method relies on the solution of the constrained equation of motion in the 
presence of frictional forces, eq 3 , which reduces to the particular form given by eq 11 for small incremental perturbations in the absence of inertial effects. Adopting realistic chain conformational and geometrical characteristics, with mathematical methods widely employed in chain statics, the matrix equation, eq 29, is derived and solved with the aim of visualizing the kinematics of rotational isomerization in various $M C$ generated chains. Applications, advantages, and future extensions of the approach may be summarized as follows:

1. For a known original configuration it is possible to follow up the most probable response of the macromolecule to an externally monitored perturbation of one or more degrees of freedom. Here a bond dihedral angle was rotated and the accompanying change in all other generalized coordinates was observed. To infer some general information on the kinematics of conformational rearrangements, an ensemble of $\mathrm{MC}$-generated original configurations were explored. However, the method may be particularly useful in macromolecules with welldefined equilibrium configurations such as proteins having a unique native state. The precise response of the specific structure to site perturbations and the existence and strength of correlations between various degrees of freedom may be identified in known structures, as illustrated in the examples of Figure $2 a-c$.

2. The type and extent of coupling between bond torsions depend on the interplay between intramolecular and intermolecular effects. In the absence of internal barriers to rotation, first neighbors undergo the largest counterrotation in response to a bond isomerization. As internal barriers become operative, the response of the second neighbors takes over that of the first one, as shown in Figure 3b. Insofar as the absolute changes in bond dihedral angles are concerned, distortion up to $\sim 50^{\circ}$ occurs from the part of the first two neighbors in the absence of internal barriers. The amplitude of these distortions is gradually attenuated with increasing torsional barriers although the distribution of $\left\langle\left|\Delta \varphi_{i}\right|\right\rangle$ about the rotating bond preserves its shape, as demonstrated in Figure 4. Yet, this distribution which was obtained for tetrahedrally bonded chains may change with the backbone geometry, as suggested by the $\mathrm{BD}$ simulations of polyisoprene by Ediger and Adolf. ${ }^{9}$

3. Effective activation energies for bond isomerization in various media are obtainable by the presently developed model. In dilute solution, apparent activation energies of about one single barrier height for bond torsion underlie local conformational rearrangements as demonstrated in Figure 6a,b, whereas with increasing environmental resistance, coupled torsion of two or more bonds becomes necessary and the effective activation energy during bond isomerization definitely exceeds that of single bond transition. Therefore, in a highly viscous environment like the bulk state above but close to the glass transition temperature, the apparent activation energy is significantly marked by intermolecular effects.

4. A correlation length covering up to \pm 8 neighboring atoms is observed when atomic displacements accompanying bond rotations are considered. Atoms of the rotating bond translate on the average by $\sim 0.7 \AA$ during isomerization, in conformity with $\mathrm{BD}$ simulations. The apparent shorter-range coupling in BD simulations is attributed to the superimposition of several modes of relaxation preventing one from dis- tinguishing the net effect of one single bond rotation.

5. The conformational energy $V$ approximates in the present work the torsional potential of polyethylene-like chains, with preference for the trans isomeric state over the gauche ${ }^{ \pm}$states. $V$ may be deliberately selected so as to account for other types of the intramolecular interactions, leading, for example, to helices as a result of preferential occurrence of gauche states. Additionally, the mechanism of relaxation in response to bond isomerization may be studied in a variety of chemical structures with different intrinsic conformational characteristics.

6. A broad distribution of small-amplitude changes in bond dihedral angles is predicted by the theory in the close neighborhood of a rotating bond. This suggests that the 2-d NMR observation of small fluctuations in bond rotational angles by Spiess and collaborators ${ }^{16}$ does not necessarily imply the absence of jumps between rotational isomeric states but appears as a consequence of the cooperative motion of chain segments for accommodating bond torsional transitions.

Acknowledgment. Partial support from Bogazici University Research Funds Project 94P0002 is gratefully acknowledged.

\section{Appendix}

Here we present a brief summary of the expressions obtained in previous work ${ }^{2,4}$ for the derivatives appearing in eq 11. The reader is referred to the original papers for details of derivation. Following the conventional treatment of chain conformational statistics, ${ }^{5,18}$ it proves mathematically convenient to define local bond-based coordinate systems $x_{i} y_{i} z_{i}$ appended to each backbone bond $i, 1 \leq i \leq n$. The $x_{i}$ axis is in the direction of the $i$ th bond, and the $y_{i}$ axis is in the plane of bonds $i$ and $i-1$, making an acute angle with the prolongation of bond $i-1$. The $z_{i}$ axis completes a right-handed frame. The transformation from frame $i+1$ into frame $i$ is performed with the use of the transformation matrix $\mathbf{T}_{i}$. The position vector $\mathbf{r}_{i}$ of the $i$ th atom relative to the local frame $x_{0} y_{0} z_{0}$ affixed to the first atom of the chain is evaluated from

$$
\mathbf{r}_{i}=\left[\begin{array}{ll}
\mathbf{E} & 0
\end{array}\right]\left[\prod_{k=1}^{i-1} \mathbf{G}_{k}\right]\left[\begin{array}{l}
\mathbf{1} \\
1
\end{array}\right]
$$

where 1 is the local bond vector $\operatorname{col}(l 00)$ for bonds of length $l, \mathbf{E}$ is the identity matrix of order 3 , and $\mathbf{G}_{i}$ is the conventional generator matrix for determining $\mathbf{r}_{i}{ }^{18}$ The summation starting from index 1 includes the rotation of the first bond, which represents one Euler rotation. The passage to absolute position vectors $\mathbf{R}_{\text {. }}$ is readily established by the identity

$$
\mathbf{R}_{i}=\mathbf{T}(\Psi) \mathbf{T}(\boldsymbol{\Phi}) \mathbf{r}_{i}+\mathbf{R}_{0}
$$

where $\mathbf{T}(\Psi)$ and $\mathbf{T}(\Phi)$ are the transformation matrices accounting for the orientation of the first bond of the chain in space.

Adopting this framework, the first term in the first line of eq 11 is obtained from

$$
\frac{\delta t}{2 \xi} \frac{\partial \delta W_{\xi}}{\partial \delta \varphi_{m}}=\sum_{j=1}^{n-1} u_{m j} \delta \varphi_{j}+p_{m} \delta \Psi+w_{m} \delta \Phi+\mathbf{v}_{m} \cdot \delta \mathbf{R}_{0}
$$

where the coefficients of the incremental generalized 


\section{Bahar et al.}

coordinates are defined as

$$
\begin{gathered}
p_{m}=\sum_{i=m+1}^{n}\left(\mathbf{a}_{i m} \cdot \mathbf{D} \mathbf{r}_{i}\right) \\
w_{m}=\sum_{i=m+1}^{n}\left(\mathbf{a}_{i m} \cdot \mathbf{B} \mathbf{r}_{i}\right) \\
\mathbf{v}_{m}=\mathbf{T}(\Psi) T(\Phi) \sum_{i=m+1}^{n} \mathbf{a}_{i m} \\
u_{m j}=\sum_{i=k+1}^{n} \mathbf{a}_{i m} \cdot \mathbf{a}_{i j}
\end{gathered}
$$

with the summation index $k=\max (m, j)$, and

$$
\mathbf{a}_{i j} \equiv\left[\prod_{k=0}^{j-1} \mathbf{T}_{k}\right] \mathbf{A T}_{j}\left[\begin{array}{ll}
\mathbf{E} & 0
\end{array}\right]\left[\prod_{k=j+1}^{i-1} \mathbf{G}_{k}\right]\left[\begin{array}{l}
\mathbf{1} \\
1
\end{array}\right]
$$

where $\mathbf{A}, \mathbf{B}$, and $\mathbf{C}$ are unitary matrices yielding the derivatives of the transformation matrices according to the relationships

$$
\begin{aligned}
\frac{\partial \mathbf{T}_{j}}{\partial \varphi_{j}}=\mathbf{A} \mathbf{T}_{j} ; \quad \frac{\partial \mathbf{T}(\Phi)}{\partial \Phi}=\mathbf{T}(\Phi) \mathbf{B} ; & \frac{\partial \mathbf{T}(\Psi)}{\partial \Psi}=\mathbf{T}(\Psi) \mathbf{C}
\end{aligned}
$$

and finally $\mathbf{D}$ is defined as $\mathbf{D}=\mathbf{T}(\Phi)^{\mathrm{T}} \mathbf{C T}(\Phi)$, the
Macromolecules, Vol. 28, No. 4, 1995

superscript $\mathrm{T}$ indicating the transpose. Expressions derived similarly for the derivatives appearing in the second line of eq 11 are explicitly given in ref 2 .

\section{References and Notes}

(1) Helfand, E. Science 1984, 226, 647.

(2) Bahar, I.; Erman, B.; Monnerie, L. Macromolecules 1992, 25 , 6309.

(3) Bahar, I.; Erman, B.; Monnerie, L. Macromolecules 1992, 25, 6315.

(4) Erman, B.; Bahar, I. In Polymer Solutions, Blends, and Interfaces; Noda, I., Rubingh, D. N., Eds.; Elsevier Science Publishers B.V.: Amsterdam, 1992; p 197.

(5) Flory, P. J. Statistical Mechanics of Chain Molecules; Interscience: New York, 1969.

(6) Helfand, E.; Wasserman, Z. R.; Weber, T. A. J. Chem. Phys. 1979, 70, 2016.

(7) Helfand, E.; Wasserman, Z. R.; Weber, T. A. Macromolecules $1980,13,526$.

(8) Adolf, D. B.; Ediger, M. D. Macromolecules 1991, 24, 5834.

(9) Ediger, M. D.; Adolf, D. B. Adv. Polym. Sci. 1994, 116, 207.

(10) Goldstein, H. Classical Mechanics; Addison-Wesley: Reading, MA, 1980.

(11) Fixman, M. J. Chem. Phys. 1965, 42, 3831.

(12) Skolnick, J.; Helfand, E. J. Chem. Phys. 1980, 72, 5489.

(13) Ryckaert, J. P.; Bellemans, A. Chem. Phys. Lett. 1975, 30, 123.

(14) Morawetz, H. Science 1979, 203, 405.

(15) Bahar, I.; Erman, B. Macromolecules 1987, 20, 2310.

(16) Schaefer, D.; Spiess, H. W.; Suter, U. W.; Fleming, W. W. Macromolecules 1990, 23, 3431 .

(17) Adolf, D. B.; Ediger, M. D. Macromolecules 1992, 25, 1074.

(18) Flory, P. J. Macromolecules 1974, 7, 381.

MA940996W 